\title{
What is the contribution of iEEG as compared to other methods to cognitive neuroscience?
}

\author{
Jing Liu ${ }^{1}$, Gui Xue ${ }^{2,3 *}$
}

1. Department of Psychology, The State Key Laboratory of Brain and Cognitive Sciences, University of Hong Kong, Hong Kong, China

2. State Key Laboratory of Cognitive Neuroscience and Learning, Beijing Normal University, Beijing, China

3. IDG/McGovern Institute for Brain Research, Beijing Normal University, Beijing, China

Note: This chapter is forthcoming in the book, Intracranial EEG for Cognitive Neuroscience

\begin{abstract}
Intracranial electroencephalography (iEEG) enables us to record and modulate macro and micro neuronal responses in both cortical and subcortical regions of the human brain with high spatial and temporal precision, offering significant methodological advantages over other non-invasive imaging and stimulating technologies. Leveraging these technical strengths of iEEG, in combination with sophisticated multivariate analytical approaches, researchers have obtained unprecedented insights into many long-standing problems in cognitive neuroscience. This chapter aims to illustrate these contributions, focusing on human memory. In particular, we describe how iEEG could advance our understanding of (1) the dynamic and transformative nature of short-term and long-term memory representations; (2) the role of hippocampal highfrequency neural activities, especially ripple activities, in memory formation, consolidation, and retrieval; (3) the information coding scheme of single-neuronal activity in the hippocampus and other brain regions; and (4) the common and different neural mechanisms between humans, primates and rodents. Moreover, we briefly discuss how iEEG studies can contribute to developing the state-of-the-art brain-computer interface and closed-loop brain stimulation. We conclude by summarizing the strengths and limitations of the iEEG method and providing practical guidance on how to choose between iEEG and other methods.
\end{abstract}




\section{Introduction}

It is generally accepted that human brain functions, such as perception, attention, decision, learning, and memory, are supported by dynamic and interactive neuronal activities. At the microscopic level, neuronal activities can be well characterized by two main types of electrical activity, i.e., neuronal spikes and postsynaptic potentials. The neuronal spikes are the action potentials that travel along the axons of the neuron in an all-or-none form, with a very short duration of approximately one millisecond. Multiple spikes, which result in a spiking train and a highly intricate temporal pattern, serve as the code of brain information (Zeldenrust et al. 2018; Azarfar et al. 2018). Because of the brief timing and opposite electrical currents flow in the intracellular and extracellular spaces, the neuronal spikes can only be recorded with a sensor near enough to the neuron. In contrast to the spikes, the postsynaptic potentials (PSPs) are changes of the potentials in the membrane of the neurons (Purves et al. 2001), which have a longer duration ranging from tens- to hundreds- of milliseconds. The resultant electrophysiological signals, termed the local field potential (LFP), could propagate and thus be recorded both within and outside the skull.

For ethical reasons, most human studies rely on non-invasive brain imaging techniques, including electroencephalogram (EEG), magnetoencephalography (MEG), functional nearinfrared spectroscopy (fNIRS), functional magnetic resonance imaging (fMRI), and positron emission tomography (PET). These methods provide either an indirect (e.g., fNIRS, PET, or fMRI) or a coarse (e.g., EEG and MEG) measure of neuronal responses. The intracranial EEG (iEEG) recordings, which are mainly applied in drug-resistant epilepsy patients for clinical purposes, provide a rare opportunity to direct record neuronal responses inside the human brain (see also chapter 2 for a detailed description of epilepsy patients and electrodes implantation). iEEG could record both LFP and neuronal spikes with macroelectrodes and microelectrodes, respectively. Specifically, there are two types of macroelectrode recordings, i.e., Electrocorticography (ECoG) and Stereoelectroencephalography (sEEG). Whereas ECoG recordings place electrodes on the surface of the brain, the sEEG uses depth electrodes perpetrating the brain to target deep brain structures. Similarly, using microelectrode arrays or microwires on the tips of the depth electrodes, iEEG can be used to record single-unit activities from the surface and deep structure of the brain (see also chapters 19 and 20 for a detailed description of iEEG signal characteristics). Compared with the non-invasive brain imaging methods, iEEG could offer several critical methodological advantages to better uncover the neuronal mechanisms of human cognition. In this chapter, we will summarize these methodological advantages and discuss how they could be leveraged to address long-standing problems in cognitive neuroscience, with a focus on learning and memory. We will conclude by providing practical guidance on how to choose between iEEG and other non-invasive methods.

\section{The methodological advantages of iEEG}

\section{High spatial-temporal resolution}

fMRI and PET are well-known for their high spatial resolution (1-3mm) and whole-brain coverage. However, they indirectly measure neuronal responses (e.g., blood flow or metabolism rate), with the temporal resolution at the level of seconds. The temporal resolution of EEG/MEG ravels the iEEG, but their spatial resolution is largely degraded by the volume conduction. The irreversible problem in untangling the sources that contribute to the observed EEG/MEG signals 
further impedes research from precisely localizing the origins of signals, especially those from deep brain areas.

The iEEG has both high spatial and temporal resolutions. The grid and/or strip electrodes placed on the subdural of the cortex are $\sim 2 \mathrm{~mm}$ in diameter and covers $\sim 4 \mathrm{~mm}^{2}$ in the cortical surface. Meanwhile, each depth electrode has about 6-20 contacts, with a diameter of $\sim 1 \mathrm{~mm}$ and a length of $\sim 2 \mathrm{~mm}$ for each contact. Given the diameters of the electrodes and their adjacency to neurons, it is assumed that iEEG could record the local field potentials from neural populations within a few millimeters (Engel et al. 2005; Mukamel and Fried 2012). The microwire electrodes used for single-unit recordings are about $40 \mu \mathrm{m}$ in diameter (Kreiman 2007), with a spatial resolution of less than $1 \mathrm{~mm}$. In addition, with a sampling rate ranging from $\sim 1000-3000 \mathrm{~Hz}$ for macroelectrodes and $\sim 30 \mathrm{kHz}$ for microelectrodes, $\mathrm{iEEG}$ is able to observe the rapid change of local neural activities at a millisecond to submillisecond level.

In summary, due to the high spatial and temporal resolution, iEEG is one of the best methods to characterize the dynamic changes of neural processes and representations across time and brain regions.

\section{High signal-to-noise ratio}

Since iEEG could directly record the neuronal responses around its origin, it could yield a greater signal-to-noise ratio (SNR). In contrast, the EEG and MEG record brain signals at the scalp, which is far away from the origins. In addition, the iEEG recordings are less sensitive to environmental noise and artifacts. For example, the fMRI and MEG are both sensitive to the magnetic field change in the recording environments. A slight head movement could result in motion artifacts and introduce challenges in an accurate spatial registration for fMRI and MEG. For both EEG and MEG, body movement, eye blink, or muscle tension could introduce external noise into the recorded signals. In contrast, the iEEG is less sensitive to movement or magnetic field changes. As a result, its signal-to-noise ratio (SNR) is about 20-100 times higher than scalp EEG (Ball et al. 2009). The high SNR of iEEG helps us distinguish the task-related neural activities more meticulously.

\section{High-frequency activity}

High-frequency activity $(50 \mathrm{~Hz}-150 \mathrm{~Hz})$ is the neural oscillations generated by the local neuronal population (Ray et al. 2008), which is characterized by high frequency and low amplitude activities with high temporal resolution. Given the local properties of high-frequency activity and the skull acting as the low-pass filter, these high-frequency activities cannot be easily detected by non-invasive imaging methods, including fMRI, PET and EEG. Although the MEG has been believed to contain neural activities up to about $100 \mathrm{~Hz}$ (Hansen et al. 2010), its signal-to-noise ratio for high-frequency activities is lower than iEEG in general. This makes the iEEG the optimal way to unveil how high-frequency activities during both online (wake) and offline (sleep) periods.

\section{Direct electrical stimulation of the human brain}

In addition to recording the neural activities from the brain, iEEG electrodes can also be used to deliver precise electric pulses to specific brain areas. As compared with the transcranial stimulation systems, such as tDCS/tACS and TMS, the direct brain stimulation by iEEG 
electrodes enjoys greater specificity. For example, it could target the medial temporal lobe (MTL), a region that is critical for declarative memory (Jacobs et al. 2016), or the orbitofrontal cortex that is involved in mood state (Rao et al. 2018). In cognitive studies, different stimulation parameters have been applied. In general, the electrical pulses are with a width of $100-300 \mu \mathrm{s}, \mathrm{a}$ current amplitude of $0.5-6 \mathrm{~mA}$, and stimulation frequency can be either at a low-frequency range (e.g., 2-5Hz) or at high frequencies (e.g., $50 \mathrm{~Hz}, 60 \mathrm{~Hz}$, or 130Hz) (Suthana et al. 2018).

Table 1. Summary of the methodological advantages of $i E E G$ as compared to common noninvasive imaging methods

\begin{tabular}{|l|l|l|l|l|}
\hline Imaging features & iEEG & MEG & EEG & fMRI \\
\hline $\begin{array}{l}\text { Neural } \\
\text { electrophysiological } \\
\text { activities }\end{array}$ & Yes & Yes & Yes & No \\
\hline Temporal resolution & $<1 \mathrm{~ms}$ & $<1 \mathrm{~ms}$ & $<1 \mathrm{~ms}$ & $\sim 5 \mathrm{~s}$ \\
\hline Spatial resolution & $<1 \mathrm{~cm}$ & $\sim 10 \mathrm{~cm}$ & $\sim 10 \mathrm{~cm}$ & $\sim 1 \mathrm{~mm}$ \\
\hline $\begin{array}{l}\text { High-frequency } \\
\text { oscillations }\end{array}$ & $\begin{array}{l}\text { Up to } \\
150 \mathrm{~Hz}\end{array}$ & $<100 \mathrm{~Hz}$ & $<70 \mathrm{~Hz}$ & No \\
\hline Spike activities & Yes & No & No & No \\
\hline Signal-to-noise ratio & High & Relatively low & Relatively low & High \\
\hline Deep brain activities & Yes & No & No & Yes \\
\hline Direct brain stimulation & Yes & No & No & No \\
\hline
\end{tabular}

\section{Characterize the dynamic and transformative nature of neural representations}

The information processing in the brain is highly dynamic, showing fast and prominent changes in neural processes and representations in different brain regions. Intracranial EEG recordings could directly measure brain activities from a wide range of frequencies from $0.5 \mathrm{~Hz}$ up to $150 \mathrm{~Hz}$ with a high temporal resolution, which is ideal for characterizing the dynamic information processing at a fine spatio-temporal scale. Using this approach, many studies have uncovered the dynamic and transformative nature of neural representations, particularly during short-term and long-term memory, which have profoundly changed our perspective of human memory.

For example, the classic persistent activity model postulates that the initially formed neural patterns persist during the short-term maintenance period (Fuster and Alexander 1971; Curtis and D'Esposito 2003). With high temporal resolution, a recent iEEG study showed that the neural patterns of visual items experienced rapid changes from visual formats to abstract semantic formats, and the latter was more robustly maintained during short-term memory (Liu et al. 2020) (Fig. 1A-B). This study further revealed that the neural representations of memory items were 
dynamically reactivated during the maintenance period and coupled to the phase of hippocampal low-frequency activities.

Regarding the maintenance of multiple items in the short-term memory, the theta-gamma coding scheme posits that individual items are represented by the synchronized activation of different subsets of neural assemblies at the gamma frequency range and the phase of theta oscillations. In particular, the hippocampal theta oscillation acts as a glue that tightly links items to its different phases in support of short-term memory maintenance (Lisman and Jensen 2013). Using EEG/MEG, studies have found that the theta-gamma coupling during the short-term memory maintenance period (Sauseng et al. 2009; Fuentemilla et al. 2010; Kamiński et al. 2011). On the other hand, fMRI studies have shown that the short-term memory information is stored in distributed brain regions, including the sensory cortex and higher-order brain regions, e.g., the frontal and parietal lobes (Bettencourt and Xu 2016; Christophel et al. 2017). However, the dynamic interactions among these regions and the unique role of the hippocampus in thetagamma coding cannot be addressed using non-invasive recordings. Using iEEG recordings, studies showed that the theta-gamma coupling is not only observed in the hippocampus (Axmacher et al. 2010; Leszczyński et al. 2015), but also in multiple neocortical sites (Bahramisharif et al. 2018) during multi-item short-term memory (Fig.1C). It further shows that the interactions between hippocampal and cortical regions increase with working memory loads (Boran et al. 2019). Leveraging the high spatiotemporal resolution, several iEEG studies have revealed the directional interactions between MTL and cortical regions at various processing stages (Axmacher et al. 2008b; Chaieb et al. 2015; Johnson et al. 2018; Dimakopoulos et al. 2021). Specifically, the hippocampus replay preceded that in the sensory cortex during the maintenance period, whereas a reversed pattern was observed during the encoding period (Dimakopoulos et al. 2021).

Intracranial EEG studies could also shed light on the subprocesses of memory formations at a higher spatial resolution. For example, ERP analysis of EEG/MEG signals revealed that a late positive complex (LPC) component starting from 500 ms post-stimulus onset, and an FN400 component with a peak around $400 \mathrm{~ms}$, are both associated with memory retrieval (Woodruff et al. 2006; Voss and Federmeier 2011). Using iEEG, Fernández and colleagues found that the memory-related ERPs included at least two different subprocesses that were executed sequentially (Fernández et al. 1999). The intracranial ERPs diverged between subsequent remembered and forgotten items from about $300 \mathrm{~ms}$ in the anterior parahippocampal cortex, followed by the reversed polarity in the hippocampus from about $500 \mathrm{~ms}$ post-stimulus onset, suggesting a dynamic interaction between MTL subregions.

Intracranial EEG studies could provide a detailed picture of the transformative nature of longterm memory representations. Guided by Tulving's mental time traveling perspective of episodic memory (Tulving 2002), extant studies have provided evidence that retrieval involves the reinstatement of neural activation during encoding, which enables humans to vividly reexperience the past (Staresina et al. 2012, 2016; Ritchey et al. 2013; Yaffe et al. 2014). Nevertheless, there is now increasing neural evidence to suggest that memory is rather a constructive process (Bartlett et al. 1932; Barry and Maguire 2019) which involves a substantial transformation of neural representations. For example, an fMRI study found that item-specific encoding-retrieval similarity was not significant and lower than the neural pattern similarities within the encoding and retrieval stages (Xiao et al. 2017), suggesting the neural representations 
were transformed from encoding to retrieval. A recent iEEG study took advantage of its high temporal resolution to systematically delineate the dynamic transformation of neural representations from encoding to maintenance and retrieval period (Liu et al. 2021a) (Fig. 1D-F). It showed that neural representations of memory items were highly dynamic during encoding, evolving rapidly from visual to abstract semantic representations. Interestingly, greater encoding dynamicity predicted better subsequent long-term memory performance. After encoding, these neural representations experienced continuous transformation during maintenance and retrieval, and only abstract semantic representations are reactivated in successful long-term memory retrieval.

Recent iEEG studies have provided a more detailed description of the temporal dynamics of the hippocampus and cortical region during memory retrieval, which emphasized the role of the hippocampus in driving the cortical activities during retrieval. Specifically, The high-frequency activities (gamma band activity in the range of $40-50 \mathrm{~Hz}$ ) in the hippocampus preceded the decreased oscillatory power in the alpha/beta band in the anterior temporal lobe during memory retrieval (Griffiths et al. 2019). In the long-term memory recognition task, the reinstatement of item-context associations occurred within the first second of retrieval in the hippocampus, followed by the reinstatement of item-specific information in the lateral temporal lobe from $\sim 1 \mathrm{~s}$ to $\sim 3$ s of retrieval (Pacheco Estefan et al. 2019). The same study also revealed that the phase synchronization between the hippocampus and lateral temporal lobe preceded the item-specific reinstatement in the lateral temporal lobe.

A

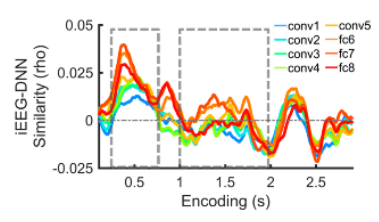

C

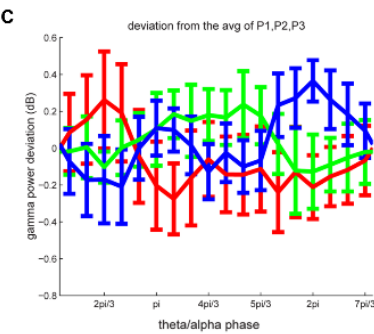

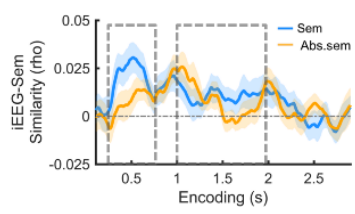
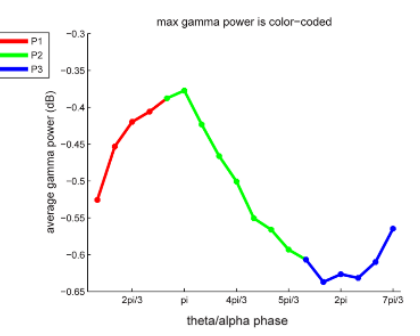

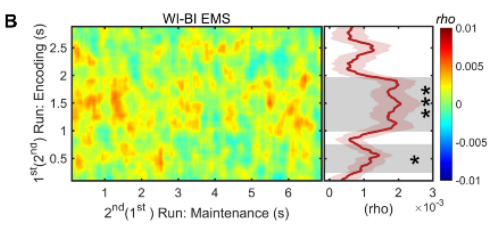

D

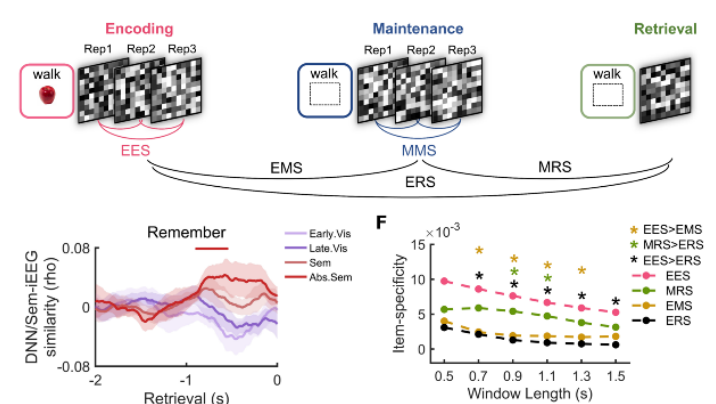

Fig. 1 Dynamic and transformative memory representations during encoding, maintenance, and retrieval. (A) Higher-order visual representations and abstract semantic representations during early and late encoding clusters, respectively. Left: visual representational formats observed in the iEEG data during encoding of visual objects via using an eight-layer visual deep neural network model (AlexNet, see Krizhevsky et al. 2012). Right: Semantic and abstract semantic formats during encoding of visual objects via using Chinese word embedding model (see Song et al. 2018). Dashed frames indicate the encoding time clusters, during which the neural activities were reinstated during the short-term maintenance period. (B) Abstract semantic representations from the late encoding cluster are better maintained in the short-term memory. $(\boldsymbol{C})$ Items learned in a sequential order were locked to different phases of low- 
frequency oscillations in the neocortex. P1/P2/P3 indicates the positions (order) of items in a three-item sequence. $(\boldsymbol{D})$ Illustration of examining the continuous representational transformation of memory items across task stages. $(\boldsymbol{E})$ Abstract semantic representations of visual items are reinstated during successful retrieval. $(\boldsymbol{F})$ Continues transformation from encoding to maintenance and retrieval. Cross-stage transformation was examined by comparing the within-stage versus between-stage item-specific pattern similarity. EES: encoding-encoding similarity; EMS: encoding-maintenance similarity; MRS: maintenance-retrieval similarity; ERS: encoding-retrieval similarity; WI: within-item similarity; BI: between-item similarity. *: $p<$ 0.05. (A-B), adapted from Liu et al. (2020). (C), adapted from Bahramisharif et al. (2018). (D$F)$, adapted from Liu et al. (2021).

\section{The role of hippocampal ripple activities in memory}

The ripple activities were first discovered in the rodent hippocampus with the frequency range between $\sim 140-200 \mathrm{~Hz}$ (O’Keefe and Nadel 1978; Buzsáki et al. 1992). They are co-incident short-lived fast oscillatory patterns that are generated by the pyramidal cell ensembled in the CA1 (Csicsvari et al. 2000). Animal studies have implicated the hippocampal sharp-wave ripples (SWRs), the co-occurrence of ripples and sharp waves, in memory consolidation (Wilson and McNaughton 1994; Diba and Buzsáki 2007; Fernández-Ruiz et al. 2019). Specifically, rodent studies have observed that the sequential firing of hippocampal places cells during spatial navigation was replayed during the post-learning wakeful rest (Wilson and McNaughton 1994; Diba and Buzsáki 2007), as well as during sleep (Nádasdy et al. 1999), in particular, slow-wave sleep stage (Lee and Wilson 2002). Strikingly, these replays were often accompanied by hippocampal SWRs. Selective disruption of hippocampal SWRs via electrical stimulation during sleep could impair memory formation (Girardeau et al. 2009; Ego-Stengel and Wilson 2010). Using a similar method to interrupt SWRs during the encoding stage, a later study also found impairment of memory formation and memory-based decision making, suggesting an important role of hippocampal SWRs in memory encoding, consolidation, and retrieval (Jadhav et al. 2012).

In light of these inspiring findings from animal studies, it is natural to ask whether there are ripples in the human hippocampus and, if so, does hippocampal ripples only support spatial memory as extensively examined in animal studies, or it also supports other types of declarative memory in humans? Due to its high frequency and deep origin, it is a challenge to confidently isolate hippocampal ripples by non-invasive imaging methods (see recent efforts by Liu and colleagues, Liu et al. 2021b). Using iEEG recordings, several studies have investigated the functional role of hippocampal ripples in humans. First, iEEG studies have observed ripples in the human hippocampus (Fig. 2A-B), although at a lower frequency band $(80-140 \mathrm{~Hz})$ than that found in the rodent hippocampus (Clemens et al. 2007; Axmacher et al. 2008a). Hippocampal ripples are found during an attention-demanding cognitive task, autobiographic memory recall, wakeful rest, and sleep (Fig. 2C), and the duration, rate, and peak frequencies are surprisingly consistent across states (Chen et al. 2021; Norman et al. 2021). Notably, the ripple rate during non-rapid eye movement (NREM) sleep is higher than during other task states and sleep stages. Ripple rate is also higher in the CA1 than CA2 and CA3 (Fig. 2D). The coincidence of individual ripples between every two hippocampal channels decreased sharply with increasing distance (Jiang et al. 2019; Norman et al. 2021), suggesting a local nature of the hippocampal ripples. 
Second, iEEG studies have shed light on the functional role of ripples in memory formation and consolidation. For example, several iEEG studies have associated ripple activities during sleep with post-sleep performance. For example, the number of ripples that occurred in the rhinal cortex instead of the hippocampus predicts memory performance during the post-sleep stage (Axmacher et al. 2008a). Using representational similarity analysis, an iEEG study further revealed that the replay of memory representations was time-locked to hippocampal ripples during the NREM sleep (Zhang et al. 2018) (Fig. 2E), and predicted subsequent long-term memory. These results suggest that hippocampal ripples contribute to memory consolidation via memory replay. In addition to memory consolidation, emerging human iEEG studies have shown that ripples during wakeful state predict successful encoding (Norman et al. 2019) and memory retrieval (Kucewicz et al. 2014; Vaz et al. 2019; Norman et al. 2021). During encoding, hippocampal ripple rates were significantly higher for novel stimuli than that for familiar stimuli, and higher ripple rates predicted better subsequent memory performance (Norman et al. 2019; Henin et al. 2021). During memory retrieval, hippocampal ripples modulated the high-frequency activities in the neocortex, which supported memory reinstatement (Vaz et al. 2019; Norman et al. 2019; Sakon and Kahana 2021). Together, these studies highlight an important role of ripples during both wakefulness and sleep in memory (Joo and Frank 2018).

Third, several iEEG studies have started to uncover the underlying mechanisms of hippocampal ripples in triggering cortical reinstatement. For example, an iEEG study found that ripples were locked to the phase of delta oscillation $(0.5-4 \mathrm{~Hz})$ during sleep within the hippocampus (Axmacher et al. 2008a), resembling the coupling between the hippocampal ripple and neocortical slow oscillation (SO). By simultaneously recording MTL ripples using iEEG and slow-wave oscillations using scalp EEG, one study showed that MTL ripples and spindle activities were both time-locked to the upstate of neocortical SOs (Clemens et al. 2007). The neocortical SO-spindle coupling also coordinates the MTL ripples during NREM sleep. In turn, MTL ripples mediate the information flow from the MTL to the neocortex (Helfrich et al. 2019). Consistently, another iEEG study found that neocritical spindle occurred earlier than the hippocampal spindles, followed by the hippocampal SWRs, especially for the long but not short ripples (Ngo et al. 2020) (Fig. 2F-G). These results suggest a top-down control from the neocortex to the hippocampus via sleep spindles, which potentially initiates the memory replay in the hippocampus. The hippocampal ripples then further drive the neocortical reinstatement of memory content. 
A

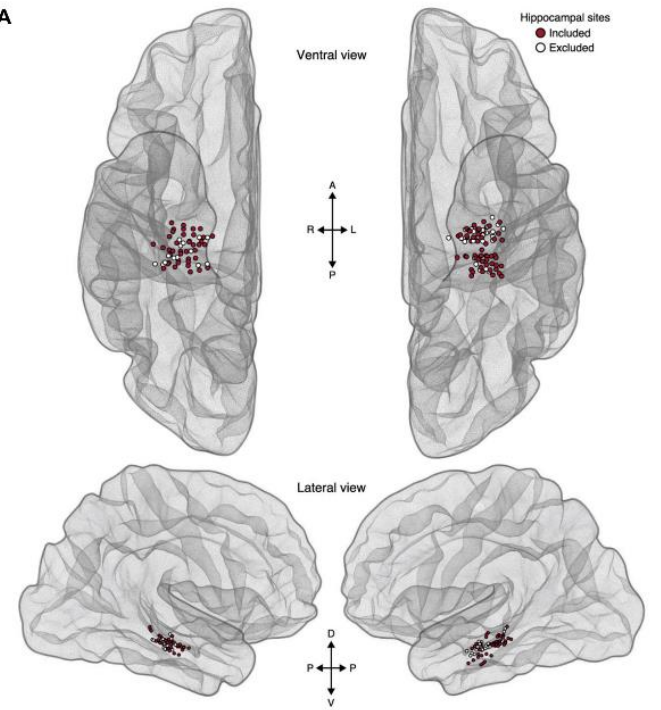

E
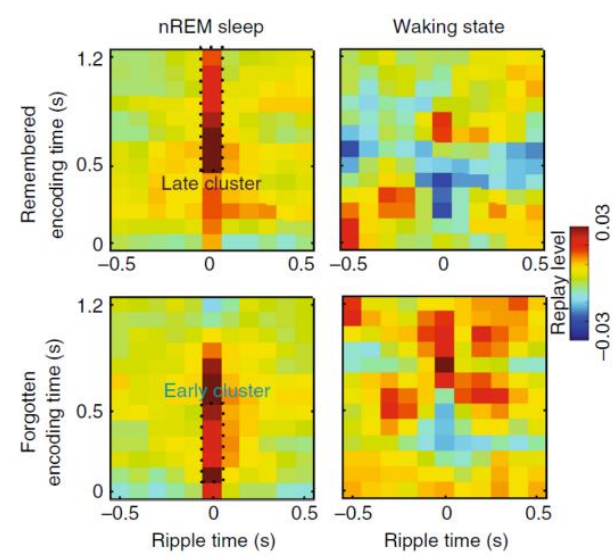

B
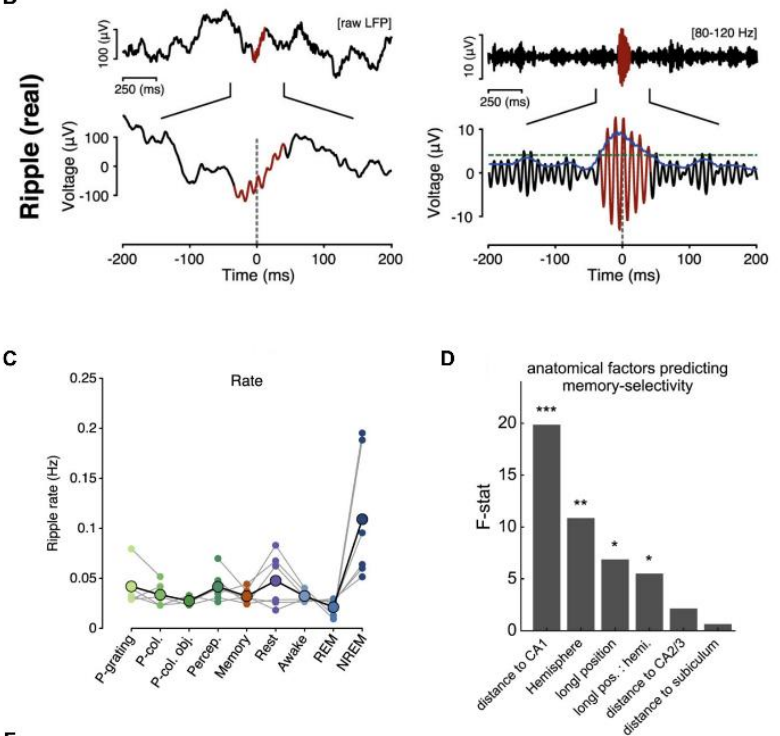

$\mathbf{F}$
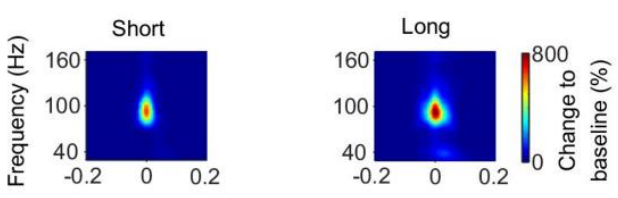

G

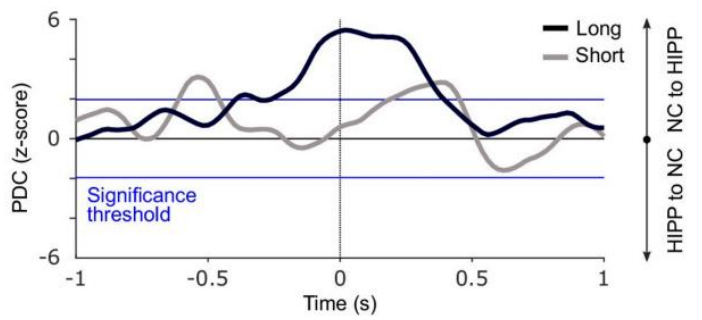

Fig. 2 Hippocampal ripple and memory reinstatement. (A) Depiction of hippocampal electrodes in epilepsy patients. (B) Example of ripple activities recorded from hippocampal electrodes. $(\boldsymbol{C})$ Ripple rates across awake tasks and sleep stages. $(\boldsymbol{D})$ the effect of anatomical locations on ripple rates in memory-selective tasks. (E) Memory replay during NREM sleep is precisely locked to ripples. $(\boldsymbol{F})$ ripple activities with short (mean \pm SEM: duration $=0.046 \pm$ 0.001 s) and long durations (mean \pm SEM: duration $=0.059 \pm 0.002 s$ ). $(\boldsymbol{G})$ Directional interactions from neocortex to hippocampus during the occurrence of ripples, especially long ripples. PDC: partial directed coherence in the 12-16Hz spindle range. NC: neocortex; HIPP: hippocampus; Positive PDC value indicates the information flowing from NC to HIPP and vice versa for negative PDC values. (A-C) adapted from Chen et al. (2021); (D) adapted from Norman et al. (2021); (E) adapted from Zhang et al. (2018); (F-G) adapted from Ngo et al. (2020).

\section{The information coding scheme of human single-neuronal activities}

Using microelectrodes, iEEG recordings are capable of capturing signals from single neurons, providing direct observation of information processing at the cellular level. Nevertheless, due to technical and ethical challenges, it is extremely difficult to record single neuronal activity in the 
human brain. Several studies have seized this rare opportunity to uncover the information coding scheme of single-neuron activities in humans. In particular, a series of studies have examined the role of MTL neurons in signaling memory content, temporal context, and novelty, as well as in supporting memory formation and maintenance.

A classical finding from human single-unit studies is the discovery of the "concept cells", a subset of MTL neurons that are selectively firing to specific persons or items (Quian Quiroga et al. 2005; Quian Quiroga 2012). For example, a subset of neurons showed increased firing rate to all photos of Jennifer Aniston with different views or backgrounds while showing very weak or no firings to the photos of other famous actresses, landmarks, animals, or objects. More interestingly, these neurons not only fired to the visual images but also the written or auditory names of the actress, suggesting an invariant, amodal conceptual representation (Quian Quiroga et al. 2009) (Fig. 3A). These cells were initially named "Jennifer Aniston" cells and are now termed "concept cells" (Quian Quiroga 2012). The proportion of modality-invariance neurons increases from the parahippocampal and perirhinal cortex to the entorhinal cortex and peaks in the hippocampus (Quian Quiroga et al. 2009), which resembles the increased visual invariance along the ventral visual pathway in non-human primates (Serre et al. 2007).

Are these concept cells also involved in the short-term maintenance when the stimuli are no longer presented on the screen? According to animal models, one potential neural mechanism of short-term memory maintenance is the persistent activation of neurons that are initially reactivated during the perception stage (Fuster and Alexander 1971; Curtis and D'Esposito 2003). This hypothesis was tested by two independent human single-unit studies, which both showed that the concept cells in the MTL fired during the encoding phase showed continuous firing during the maintenance period (Kamiński et al. 2017; Kornblith et al. 2017). Although a similar persistent activity was found in the medial frontal cortex, it was associated with the working memory load instead of memory content. It should be noted that persistent activities are generally obtained by averaging spikes across time and trials. When examining individual trials, recent studies have found that the neuronal spikes in support working memory occurred in a sparse, transient, and dynamic manner (Lundqvist et al. 2018; Miller et al. 2018).

In addition to the concept cells, human single-unit studies have also observed cells that signal visual categories, novelty, familiarity, confidence, temporal context of memory items in the MTL and other brain regions (Rutishauser et al. 2006, 2015, 2018). For example, by separating the neuronal responses according to subjects' behavioral responses in a recognition task, one study has found two types of neurons, with one showing greater firing to the novel than familiar stimuli, whereas the other showing the opposing pattern (Rutishauser et al. 2015) (Fig. 3B). In addition, neurons in the posterior parietal cortex could signal the subjective confidence level of memory decisions regardless of familiarity (Rutishauser et al. 2018). Another study found that in a memory-based decision task, neurons in the medial frontal cortex could signal the abstract task goals according to the task demands (Minxha et al. 2020). Umbach and colleagues discovered "time cells" in the human hippocampus and entorhinal cortex, which signal the temporal positions of items within encoding/retrieval tasks and predict the temporal clustering of items during memory retrieval (Umbach et al. 2020). Another study also identified time cells in the hippocampus, which track the successive temporal information during sequential learning and replay during the post-encoding gaps (Reddy et al. 2021). A recent iEEG study had demonstrated the existence of "boundary cells" in the MTL, which fired increasingly when a boundary was 
detected. The neural state changes elicited by the boundary at the population level also predicted temporal order memory (Zheng et al. 2021).

Single-unit recordings have also contributed to the understanding of associative learning and memory. Different items in the same episodic memory would rely on overlapping neuronal networks. This is achieved by the plasticity of concept neurons, whose firing properties can be reshaped by associative learning (Ison et al. 2015; Reddy et al. 2015). For example, A single neuron firing to a specific item during the pre-learning period showed an expanded preferred response to the associated items that co-occur with the preferred items after a few trials of learning (Ison et al. 2015). Once the associative memory is formed, effective memory cues would trigger a predictive firing of MTL neurons before the re-appearance of items (Reddy et al. 2015).

A

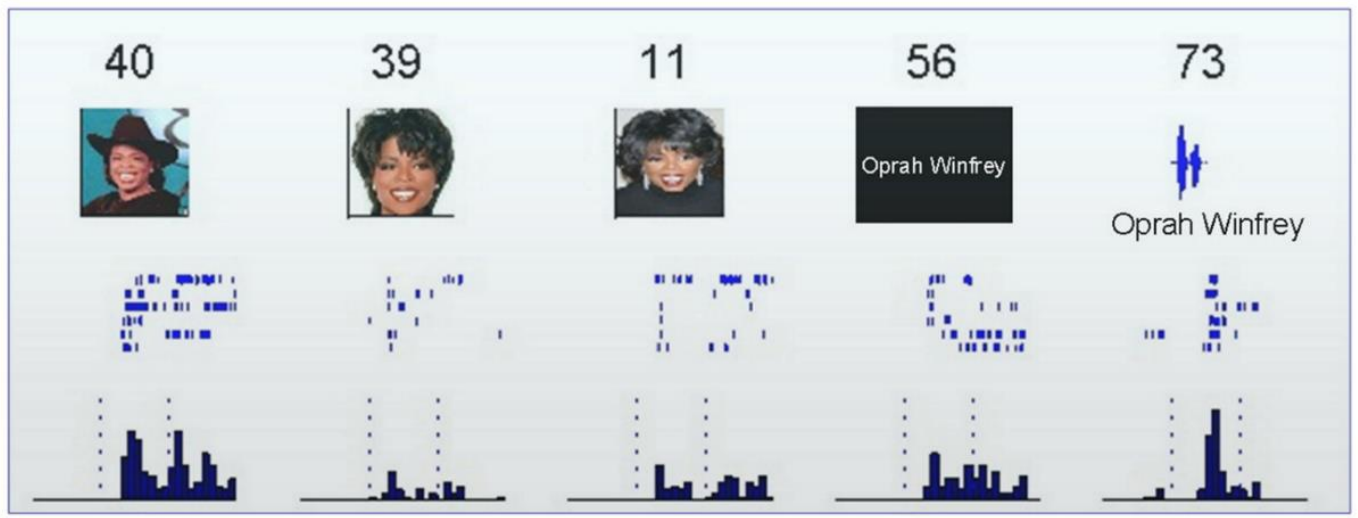

B
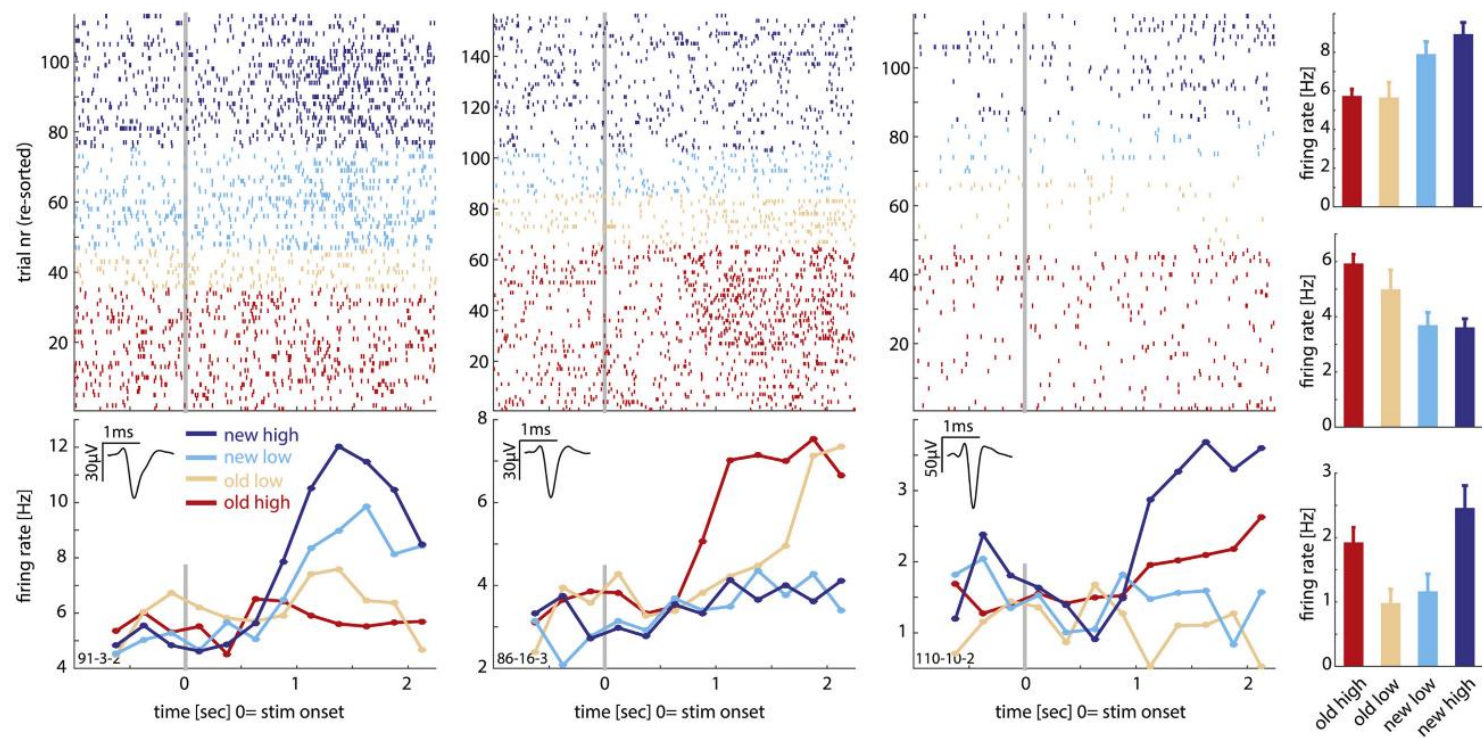

Fig. 3 Examples of memory-selective cells observed by human single-unit recordings. (A) Concept cells, which invariantly and selectively fire to specific concepts, for example, the picture or the written name of the television host Oprah Winfrey. (B) Memory-selective cells during the recognition task. first column: novelty cells; second column: familiarity cells; third column: cells 
signal confidence level; fourth column: the average firing rate of these three types of cells during a recognition task. (A) adapted from Quian Quiroga et al. (2009); (B) adapted from Rutishauser et al. (2018).

\section{The common and different neural mechanisms between humans, primates, and rodents}

Our understandings of the neuronal mechanisms of cognition have been largely advanced by animal studies. A critical question is how the findings from animal studies could inform the neural mechanisms of human cognition, given the apparent difference in brain structure and functions between animals and humans. This question is challenging to address due to different neural imaging techniques, e.g., cellular recording in animals and non-invasive imaging in humans. Human intracranial EEG associated with single-unit recordings bridges the gap, providing unprecedented insights into the shared and unique neural mechanisms between humans, non-human primates, and rodents.

Existing studies have revealed that humans and rodents share similar encoding schemes during spatial navigation, memory encoding, and consolidation. For example, A well-known finding in rodents is the grid cell in the entorhinal cortex (Hafting et al. 2005). Similarly, several iEEG studies also revealed grid-like representations in the human entorhinal cortex (Jacobs et al. 2013; Maidenbaum et al. 2018; Chen et al. 2018), and this hexadirectional modulation was associated with spatial memory performance (Maidenbaum et al. 2018; Chen et al. 2018; Jun et al. 2020). Another well-documented neural code of spatial/temporal information is the phase precession (O'Keefe and Recce 1993). Phase precession refers to the time of neurons responding to the specific locations occurring progressively earlier to the phase of local theta frequency oscillations $(\sim 5-10 \mathrm{~Hz})$, so the spatial or temporal information could be connected into an ordered sequence within theta cycles. This phenomenon was well observed in the hippocampal place cells (O'Keefe and Recce 1993) and entorhinal grid cells in rodents (Reifenstein et al. 2012). Consistently, a recent iEEG study had observed the phase precession in the human hippocampus and entorhinal cortex when participants were performing the spatial navigation tasks (Qasim et al. 2021). In addition, as described above, both rodents and human studies have implicated the hippocampal SWRs in memory consolidation (Buzsáki et al. 2013; Buzsáki 2015; Khodagholy et al. 2017; Fernández-Ruiz et al. 2019; Ngo et al. 2020; Skelin et al. 2021).

Despite these similarities, researchers have also revealed important differences between humans and non-human animals. For example, a human iEEG study showed that hippocampal theta oscillations during navigation and memory processing are at a lower frequency $(1-4 \mathrm{~Hz})$ as compared with that in rodents $(4-10 \mathrm{~Hz})$ (Watrous et al. 2013; Jacobs 2014). This slower theta frequency might enable a higher working memory capacity, according to the theta-gamma coding scheme (Lisman and Jensen 2013). Similarly, the frequency of human SWRs is also lower than that found in rodents (Clemens et al. 2007; Axmacher et al. 2008a). More importantly, it is reasonable to argue that the modality-invariance conceptual cells found in the human hippocampus (Quian Quiroga et al. 2005, 2008) might not be possible for rodents. Nevertheless, more studies are definitely required to test this hypothesis. Finally, it usually takes much longer time to train animals than humans to perform certain cognitive tasks, and this overtraining might have introduced additional cognitive and neural processes. For example, one rodent research has revealed two types of temporal order representation in the hippocampalentorhinal system: in addition to the temporal flow that is formed automatically with one-shot 
learning, there are also representations of stable event sequence which are increased in wellpracticed structured events (Tsao et al. 2018). Future studies are definitely required to address these issues.

\section{iEEG based brain-computer interface and closed-loop brain stimulation}

With the superb SNRs and high temporal resolution, the iEEG recordings could promote the development of brain-computer interfaces (BCI), a next-generation of rehabilitative technologies to restore impaired cognitive functions. For example, in a recent iEEG study, Chartier and colleagues recorded the neural activities from the human sensorimotor cortex while participants were speaking. They found the neural code that gives rise to the complex articulatory kinematics trajectories underlie fluent speech production (Chartier et al. 2018). These results were then used to develop the neural decoders that could synthesize the audible speech from the cortical activity (Anumanchipalli et al. 2019). In another instance, ECoG has been applied to measure signals from the motor cortex, which have then been used to control the prosthetic hands and arms. Compared to scalp EEG, the BCI using ECoG could achieve more accurate operation with fewer training trials (Leuthardt et al. 2004; Yanagisawa et al. 2011; Anderson et al. 2012; Romanelli et al. 2018).

As both a recording and modulation technology, iEEG could also help to develop the state-ofthe-art closed-loop brain stimulation system, which could establish a causal relationship between neural activities and behavior and also improve behavioral outcomes. Almost 70 years ago, Wilder Penfield mapped the brain functions by directly stimulating different brain areas during brain surgery (Penfield and Jasper 1954; Penfield and Roberts 2014). Although an increasing number of studies have shown that non-invasive stimulation, including transcranial current or magnetic stimulation, can modulate various brain functions, including perception (Romanska et al. 2015), attention (Li et al. 2017), decision (Xue et al. 2012), cognitive control (Cai et al. 2016), working memory and long-term memories (Marshall et al. 2004; Fregni et al. 2005; Brunoni and Vanderhasselt 2014; Lu et al. 2015), the exact mechanisms are less clear due to low spatial specificity. For example, one study directly tested the effect of the tACS via using the iEEG recordings but failed to find any effects of applied low-frequency tACS on brain rhythms (Lafon et al. 2017). Still, transcranial stimulations so far cannot directly target deep brain structures.

To circumvent these issues, researchers have applied direct electrical brain stimulation (EBS) on epilepsy patients. The studies on memories have found inconsistent results, with some studies showing memory enhancement (Suthana et al. 2012; Inman et al. 2018; Kucewicz et al. 2018), whereas others are showing memory impairment (Lacruz et al. 2010; Jacobs et al. 2016; Merkow et al. 2017; Goyal et al. 2018). The mixed results might be explained by the interacting effect of ongoing brain states and stimulation (Silvanto et al. 2008). For example, Ezzyat and colleagues showed stimulations during a state of low encoding efficacy could promote memory and otherwise could impair memory (Ezzyat et al. 2017). To address this issue, they developed a closed-loop stimulation scheme, in which the stimulation was triggered by a neural state that indicates subsequent memory forgetting. Using this novel approach, they have revealed a robust enhancement effect of hippocampal stimulation on long-term memory (Ezzyat et al. 2018) (Fig. 4). 

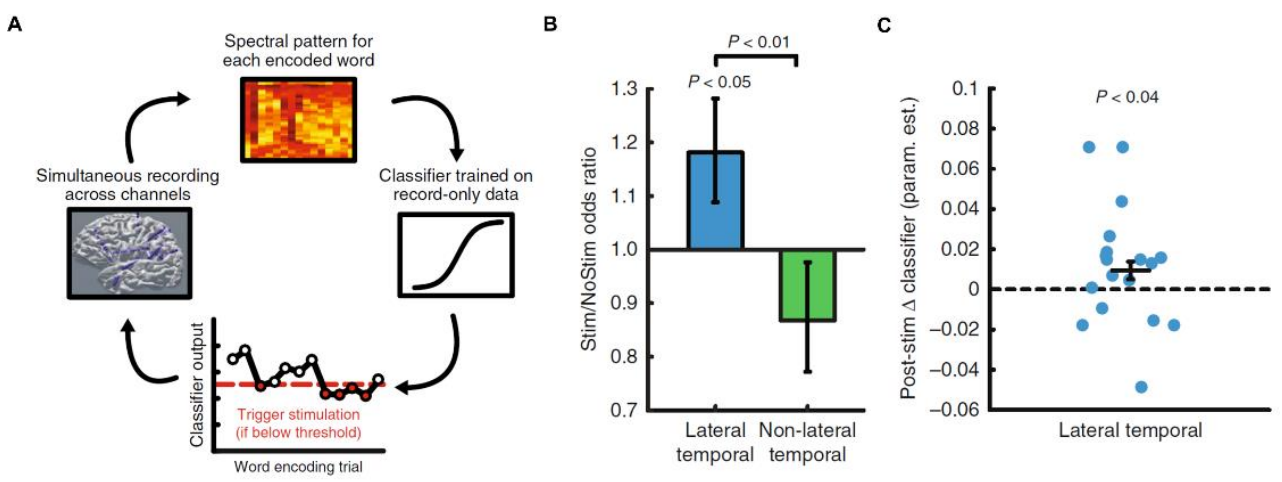

Fig. 4 Closed-loop brain stimulation using iEEG to modulate memory performance. (A) Illustration of the closed-loop stimulation. First, a classifier is trained using an independent data set. Second, the spectral decomposition analysis is performed during online recording. Third, apply the pre-trained classifier to the spectral decomposed data. Fourth, an electric pulse is triggered if the decoding evidence reaches the threshold. (B) The number of successfully recalled items in the stimulation (Stim) vs. no stimulation (NoStim) condition. The stimulation was applied to the lateral temporal lobe. $(\boldsymbol{C})$ Lateral temporal lobe stimulation increased classifier evidence during memory encoding. This figure is adapted from (Ezzyat et al. 2018).

\section{Summary and a practical guidance}

So far, we have provided an overall description of the methodological advantages of iEEG and illustrated its implications in addressing fundamental questions in cognitive neuroscience. Compared with the non-invasive brain imaging methods, the ability of iEEG to direct record single-neuron spikes and LFP with greater spatial-temporal resolution and SNRs are unprecedently rare and precious. Nevertheless, it is important to notice its limitations when planning your studies. First of all, due to ethical issues, iEEG recordings are typically performed in drug-resistant epilepsy patients for clinical purposes, which makes iEEG less accessible than other non-invasive neuroimaging methods. Second, the electrodes are usually implanted for 2-3 weeks, during which extensive physical examinations are conducted, leaving limited time windows for conducting experiments. It usually takes much longer to collect data from the required number of participants for the above reasons. Third, the electrodes are implanted to localize the seizure onset zone, thus are most likely in or near the epileptic loci. As a result, it is unlikely to get a full brain coverage of electrodes. Moreover, the signals could be smeared by the epileptic spikes, particularly when electrodes are located near the epileptic loci. Thus, iEEG is often less optimal for examining the interactions across wide brain networks. Finally, the implantation schemes are heterogeneous across patients, severely limiting the ability to compare subgroups.

Given these apparent strengths and limitations, it is vital to decide when to choose iEEG recordings over other non-invasive imaging methods. To protect patients' rights, a rule of thumb is always to choose non-invasive imaging methods when they are sufficient to address your research questions. For example, if you are interested in temporal dynamic changes of neocortical brain regions, EEG and MEG can be good options. If you are interested in localizing the brain regions where information is processed or stored, fMRI could be a good choice. Nevertheless, if you are interested in brain oscillations of deep brain regions, such as the medial 
temporal lobe or thalamus, iEEG would be a good choice. Moreover, the patients are usually in a less optimal state, cognitively and emotionally, and fatigue is a common symptom in epilepsy (Kwon et al. 2017) (see also chapter 3 for a detailed description of the cognitive status of epilepsy patients). Therefore, it is important to avoid tasks that are either cognitively challenging, may introduce intense arousal and emotional responses, or take an excessively long time (e.g., > 2 hours). Also, it is critical to avoid tasks that might induce a seizure. Finally, the iEEG study should be designed and conducted with potential benefits to the patients whenever possible. For example, in developing the mind-controlled robotic arms for paralyzed patients, intracranial EEG recordings with high SNRs and spatiotemporal resolution might be better than other non-invasive imaging methods. Similarly, deep brain stimulation protocols can be conducted to suppress seizures or improve cognitive functions (see also chapter 11 for a detailed description of iEEG experimental design and brain stimulation). Still, studies that could potentially localize the brain regions involved in essential brain functions, such as motor, language, memory, could be conducted to aid surgical planning. Together, iEEG studies are suggested when researchers need to record neural activities at high spatial-temporal resolutions from deep brain structures and should be conducted with careful considerations to patients.

\section{References}

Anderson NR, Blakely T, Schalk G, et al (2012) Electrocorticographic (ECoG) correlates of human arm movements. Exp Brain Res 223:1-10. https://doi.org/10.1007/s00221-0123226-1

Anumanchipalli GK, Chartier J, Chang EF (2019) Speech synthesis from neural decoding of spoken sentences. Nature 568:493-498. https://doi.org/10.1038/s41586-019-1119-1

Axmacher N, Elger CE, Fell J (2008a) Ripples in the medial temporal lobe are relevant for human memory consolidation. Brain 131:1806-1817.

https://doi.org/10.1093/brain/awn103

Axmacher N, Henseler MM, Jensen O, et al (2010) Cross-frequency coupling supports multiitem working memory in the human hippocampus. Proceedings of the National Academy of Sciences 107:3228-3233. https://doi.org/10.1073/pnas.0911531107

Axmacher N, Schmitz DP, Wagner T, et al (2008b) Interactions between Medial Temporal Lobe, Prefrontal Cortex, and Inferior Temporal Regions during Visual Working Memory: A Combined Intracranial EEG and Functional Magnetic Resonance Imaging Study. J Neurosci 28:7304-7312. https://doi.org/10.1523/JNEUROSCI.1778-08.2008

Azarfar A, Calcini N, Huang C, et al (2018) Neural coding: A single neuron's perspective. Neuroscience \& Biobehavioral Reviews 94:238-247.

https://doi.org/10.1016/j.neubiorev.2018.09.007

Bahramisharif A, Jensen O, Jacobs J, Lisman J (2018) Serial representation of items during working memory maintenance at letter-selective cortical sites. PLOS Biology 16:e2003805. https://doi.org/10.1371/journal.pbio.2003805

Ball T, Kern M, Mutschler I, et al (2009) Signal quality of simultaneously recorded invasive and non-invasive EEG. NeuroImage 46:708-716. https://doi.org/10.1016/j.neuroimage.2009.02.028

Barry DN, Maguire EA (2019) Remote Memory and the Hippocampus: A Constructive Critique. Trends in Cognitive Sciences 23:128-142. https://doi.org/10.1016/j.tics.2018.11.005

Bartlett SFC, Bartlett FC, Bartlett FC (1932) Remembering: A Study in Experimental and Social Psychology. Cambridge University Press 
Bettencourt KC, Xu Y (2016) Decoding the content of visual short-term memory under distraction in occipital and parietal areas. Nat Neurosci 19:150-157. https://doi.org/10.1038/nn.4174

Boran E, Fedele T, Klaver P, et al (2019) Persistent hippocampal neural firing and hippocampalcortical coupling predict verbal working memory load. Science Advances 5:eaav3687. https://doi.org/10.1126/sciadv.aav3687

Brunoni AR, Vanderhasselt M-A (2014) Working memory improvement with non-invasive brain stimulation of the dorsolateral prefrontal cortex: A systematic review and meta-analysis. Brain and Cognition 86:1-9. https://doi.org/10.1016/j.bandc.2014.01.008

Buzsáki G (2015) Hippocampal sharp wave-ripple: A cognitive biomarker for episodic memory and planning. Hippocampus 25:1073-1188. https://doi.org/10.1002/hipo.22488

Buzsáki G, Horváth Z, Urioste R, et al (1992) High-frequency network oscillation in the hippocampus. Science 256:1025-1027. https://doi.org/10.1126/science.1589772

Buzsáki G, Logothetis N, Singer W (2013) Scaling Brain Size, Keeping Timing: Evolutionary Preservation of Brain Rhythms. Neuron 80:751-764. https://doi.org/10.1016/j.neuron.2013.10.002

Cai Y, Li S, Liu J, et al (2016) The Role of the Frontal and Parietal Cortex in Proactive and Reactive Inhibitory Control: A Transcranial Direct Current Stimulation Study. Journal of Cognitive Neuroscience 28:177-186. https://doi.org/10.1162/jocn_a_00888

Chaieb L, Leszczynski M, Axmacher N, et al (2015) Theta-gamma phase-phase coupling during working memory maintenance in the human hippocampus. Cognitive Neuroscience 6:149-157. https://doi.org/10.1080/17588928.2015.1058254

Chartier J, Anumanchipalli GK, Johnson K, Chang EF (2018) Encoding of Articulatory Kinematic Trajectories in Human Speech Sensorimotor Cortex. Neuron 98:1042-1054.e4. https://doi.org/10.1016/j.neuron.2018.04.031

Chen D, Kunz L, Wang W, et al (2018) Hexadirectional Modulation of Theta Power in Human Entorhinal Cortex during Spatial Navigation. Current Biology 28:3310-3315.e4. https://doi.org/10.1016/j.cub.2018.08.029

Chen YY, Aponik-Gremillion L, Bartoli E, et al (2021) Stability of ripple events during task engagement in human hippocampus. Cell Reports 35:109304. https://doi.org/10.1016/j.celrep.2021.109304

Christophel TB, Klink PC, Spitzer B, et al (2017) The Distributed Nature of Working Memory. Trends in Cognitive Sciences 21:111-124. https://doi.org/10.1016/j.tics.2016.12.007

Clemens Z, Molle M, Eross L, et al (2007) Temporal coupling of parahippocampal ripples, sleep spindles and slow oscillations in humans. Brain 130:2868-2878. https://doi.org/10.1093/brain/awm146

Csicsvari J, Hirase H, Mamiya A, Buzsáki G (2000) Ensemble Patterns of Hippocampal CA3CA1 Neurons during Sharp Wave-Associated Population Events. Neuron 28:585-594. https://doi.org/10.1016/S0896-6273(00)00135-5

Curtis CE, D'Esposito M (2003) Persistent activity in the prefrontal cortex during working memory. Trends in Cognitive Sciences 7:415-423. https://doi.org/10.1016/S13646613(03)00197-9

Diba K, Buzsáki G (2007) Forward and reverse hippocampal place-cell sequences during ripples. Nat Neurosci 10:1241-1242. https://doi.org/10.1038/nn1961

Dimakopoulos V, Stieglitz L, Mégevand P, Sarnthein J (2021) Information flows from hippocampus to cortex during replay of verbal working memory items 
Ego-Stengel V, Wilson MA (2010) Disruption of ripple-associated hippocampal activity during rest impairs spatial learning in the rat. Hippocampus 20:1-10. https://doi.org/10.1002/hipo.20707

Engel AK, Moll CKE, Fried I, Ojemann GA (2005) Invasive recordings from the human brain: clinical insights and beyond. Nat Rev Neurosci 6:35-47. https://doi.org/10.1038/nrn1585

Ezzyat Y, Kragel JE, Burke JF, et al (2017) Direct Brain Stimulation Modulates Encoding States and Memory Performance in Humans. Current Biology 27:1251-1258. https://doi.org/10.1016/j.cub.2017.03.028

Ezzyat Y, Wanda PA, Levy DF, et al (2018) Closed-loop stimulation of temporal cortex rescues functional networks and improves memory. Nat Commun 9:365. https://doi.org/10.1038/s41467-017-02753-0

Fernández G, Effern A, Grunwald T, et al (1999) Real-Time Tracking of Memory Formation in the Human Rhinal Cortex and Hippocampus. Science 285:1582-1585. https://doi.org/10.1126/science.285.5433.1582

Fernández-Ruiz A, Oliva A, Oliveira EF de, et al (2019) Long-duration hippocampal sharp wave ripples improve memory. Science. https://doi.org/10.1126/science.aax0758

Fregni F, Boggio PS, Nitsche M, et al (2005) Anodal transcranial direct current stimulation of prefrontal cortex enhances working memory. Exp Brain Res 166:23-30. https://doi.org/10.1007/s00221-005-2334-6

Fuentemilla L, Penny WD, Cashdollar N, et al (2010) Theta-Coupled Periodic Replay in Working Memory. Current Biology 20:606-612. https://doi.org/10.1016/j.cub.2010.01.057

Fuster JM, Alexander GE (1971) Neuron Activity Related to Short-Term Memory. Science 173:652-654. https://doi.org/10.1126/science.173.3997.652

Girardeau G, Benchenane K, Wiener SI, et al (2009) Selective suppression of hippocampal ripples impairs spatial memory. Nat Neurosci 12:1222-1223. https://doi.org/10.1038/nn.2384

Goyal A, Miller J, Watrous AJ, et al (2018) Electrical Stimulation in Hippocampus and Entorhinal Cortex Impairs Spatial and Temporal Memory. J Neurosci 38:4471-4481. https://doi.org/10.1523/JNEUROSCI.3049-17.2018

Griffiths BJ, Parish G, Roux F, et al (2019) Directional coupling of slow and fast hippocampal gamma with neocortical alpha/beta oscillations in human episodic memory. PNAS 116:21834-21842. https://doi.org/10.1073/pnas.1914180116

Hafting T, Fyhn M, Molden S, et al (2005) Microstructure of a spatial map in the entorhinal cortex. Nature 436:801-806. https://doi.org/10.1038/nature03721

Hansen P, Kringelbach M, Salmelin R (eds) (2010) MEG: An Introduction to Methods. Oxford University Press, New York

Helfrich RF, Lendner JD, Mander BA, et al (2019) Bidirectional prefrontal-hippocampal dynamics organize information transfer during sleep in humans. Nat Commun 10:3572. https://doi.org/10.1038/s41467-019-11444-x

Henin S, Shankar A, Borges H, et al (2021) Spatiotemporal dynamics between interictal epileptiform discharges and ripples during associative memory processing. Brain 144:1590-1602. https://doi.org/10.1093/brain/awab044

Inman CS, Manns JR, Bijanki KR, et al (2018) Direct electrical stimulation of the amygdala enhances declarative memory in humans. PNAS 115:98-103. https://doi.org/10.1073/pnas.1714058114 
Ison MJ, Quian Quiroga R, Fried I (2015) Rapid Encoding of New Memories by Individual Neurons in the Human Brain. Neuron 87:220-230. https://doi.org/10.1016/j.neuron.2015.06.016

Jacobs J (2014) Hippocampal theta oscillations are slower in humans than in rodents: implications for models of spatial navigation and memory. Philos Trans R Soc Lond B Biol Sci 369:20130304. https://doi.org/10.1098/rstb.2013.0304

Jacobs J, Miller J, Lee SA, et al (2016) Direct Electrical Stimulation of the Human Entorhinal Region and Hippocampus Impairs Memory. Neuron 92:983-990. https://doi.org/10.1016/j.neuron.2016.10.062

Jacobs J, Weidemann CT, Miller JF, et al (2013) Direct recordings of grid-like neuronal activity in human spatial navigation. Nat Neurosci 16:1188-1190. https://doi.org/10.1038/nn.3466

Jadhav SP, Kemere C, German PW, Frank LM (2012) Awake Hippocampal Sharp-Wave Ripples Support Spatial Memory. Science. https://doi.org/10.1126/science.1217230

Jiang X, Gonzalez-Martinez J, Halgren E (2019) Coordination of Human Hippocampal Sharpwave Ripples during NREM Sleep with Cortical Theta Bursts, Spindles, Downstates, and Upstates. J Neurosci 39:8744-8761. https://doi.org/10.1523/JNEUROSCI.2857-18.2019

Johnson EL, Adams JN, Solbakk A-K, et al (2018) Dynamic frontotemporal systems process space and time in working memory. PLOS Biology 16:e2004274. https://doi.org/10.1371/journal.pbio.2004274

Joo HR, Frank LM (2018) The hippocampal sharp wave-ripple in memory retrieval for immediate use and consolidation. Nat Rev Neurosci 19:744-757. https://doi.org/10.1038/s41583-018-0077-1

Jun H, Bramian A, Soma S, et al (2020) Disrupted Place Cell Remapping and Impaired Grid Cells in a Knockin Model of Alzheimer's Disease. Neuron 107:1095-1112.e6. https://doi.org/10.1016/j.neuron.2020.06.023

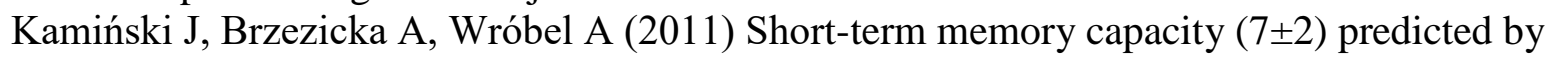
theta to gamma cycle length ratio. Neurobiology of Learning and Memory 95:19-23. https://doi.org/10.1016/j.nlm.2010.10.001

Kamiński J, Sullivan S, Chung JM, et al (2017) Persistently active neurons in human medial frontal and medial temporal lobe support working memory. Nat Neurosci 20:590-601. https://doi.org/10.1038/nn.4509

Khodagholy D, Gelinas JN, Buzsáki G (2017) Learning-enhanced coupling between ripple oscillations in association cortices and hippocampus. Science 358:369-372. https://doi.org/10.1126/science.aan6203

Kornblith S, Quian Quiroga R, Koch C, et al (2017) Persistent Single-Neuron Activity during Working Memory in the Human Medial Temporal Lobe. Current Biology 27:1026-1032. https://doi.org/10.1016/j.cub.2017.02.013

Kreiman G (2007) Single unit approaches to human vision and memory. Current Opinion in Neurobiology 17:471-475. https://doi.org/10.1016/j.conb.2007.07.005

Krizhevsky A, Sutskever I, Hinton GE (2012) ImageNet Classification with Deep Convolutional Neural Networks. In: Advances in Neural Information Processing Systems. Curran Associates, Inc. 
Kucewicz MT, Berry BM, Miller LR, et al (2018) Evidence for verbal memory enhancement with electrical brain stimulation in the lateral temporal cortex. Brain 141:971-978. https://doi.org/10.1093/brain/awx373

Kucewicz MT, Cimbalnik J, Matsumoto JY, et al (2014) High frequency oscillations are associated with cognitive processing in human recognition memory. Brain 137:22312244. https://doi.org/10.1093/brain/awu149

Kwon O-Y, Ahn HS, Kim HJ (2017) Fatigue in epilepsy: A systematic review and metaanalysis. Seizure 45:151-159. https://doi.org/10.1016/j.seizure.2016.11.006

Lacruz ME, Valentín A, Seoane JJG, et al (2010) Single pulse electrical stimulation of the hippocampus is sufficient to impair human episodic memory. Neuroscience 170:623632. https://doi.org/10.1016/j.neuroscience.2010.06.042

Lafon B, Henin S, Huang Y, et al (2017) Low frequency transcranial electrical stimulation does not entrain sleep rhythms measured by human intracranial recordings. Nat Commun 8:1199. https://doi.org/10.1038/s41467-017-01045-x

Lee AK, Wilson MA (2002) Memory of Sequential Experience in the Hippocampus during Slow Wave Sleep. Neuron 36:1183-1194. https://doi.org/10.1016/S0896-6273(02)01096-6

Leszczyński M, Fell J, Axmacher N (2015) Rhythmic Working Memory Activation in the Human Hippocampus. Cell Reports 13:1272-1282. https://doi.org/10.1016/j.celrep.2015.09.081

Leuthardt EC, Schalk G, Wolpaw JR, et al (2004) A brain-computer interface using electrocorticographic signals in humans. J Neural Eng 1:63-71. https://doi.org/10.1088/1741-2560/1/2/001

Li S, Cai Y, Liu J, et al (2017) Dissociated roles of the parietal and frontal cortices in the scope and control of attention during visual working memory. NeuroImage 149:210-219. https://doi.org/10.1016/j.neuroimage.2017.01.061

Lisman JE, Jensen O (2013) The Theta-Gamma Neural Code. Neuron 77:1002-1016. https://doi.org/10.1016/j.neuron.2013.03.007

Liu J, Zhang H, Yu T, et al (2020) Stable maintenance of multiple representational formats in human visual short-term memory. PNAS 117:32329-32339. https://doi.org/10.1073/pnas.2006752117

Liu J, Zhang H, Yu T, et al (2021a) Transformative neural representations support long-term episodic memory. Sci Adv 7:eabg9715. https://doi.org/10.1126/sciadv.abg9715

Liu Y, Mattar MG, Behrens TEJ, et al (2021b) Experience replay is associated with efficient nonlocal learning. Science 372:eabf1357. https://doi.org/10.1126/science.abf1357

Lu Y, Wang C, Chen C, Xue G (2015) Spatiotemporal Neural Pattern Similarity Supports Episodic Memory. Current Biology 25:780-785. https://doi.org/10.1016/j.cub.2015.01.055

Lundqvist M, Herman P, Miller EK (2018) Working Memory: Delay Activity, Yes! Persistent Activity? Maybe Not. J Neurosci 38:7013-7019. https://doi.org/10.1523/JNEUROSCI.2485-17.2018

Maidenbaum S, Miller J, Stein JM, Jacobs J (2018) Grid-like hexadirectional modulation of human entorhinal theta oscillations. PNAS 115:10798-10803. https://doi.org/10.1073/pnas.1805007115

Marshall L, Mölle M, Hallschmid M, Born J (2004) Transcranial Direct Current Stimulation during Sleep Improves Declarative Memory. J Neurosci 24:9985-9992. https://doi.org/10.1523/JNEUROSCI.2725-04.2004 
Merkow MB, Burke JF, Ramayya AG, et al (2017) Stimulation of the human medial temporal lobe between learning and recall selectively enhances forgetting. Brain Stimulation 10:645-650. https://doi.org/10.1016/j.brs.2016.12.011

Miller EK, Lundqvist M, Bastos AM (2018) Working Memory 2.0. Neuron 100:463-475. https://doi.org/10.1016/j.neuron.2018.09.023

Minxha J, Adolphs R, Fusi S, et al (2020) Flexible recruitment of memory-based choice representations by the human medial frontal cortex. Science 368:eaba3313. https://doi.org/10.1126/science.aba3313

Mukamel R, Fried I (2012) Human Intracranial Recordings and Cognitive Neuroscience. Annual Review of Psychology 63:511-537. https://doi.org/10.1146/annurev-psych-120709145401

Nádasdy Z, Hirase H, Czurkó A, et al (1999) Replay and Time Compression of Recurring Spike Sequences in the Hippocampus. J Neurosci 19:9497-9507. https://doi.org/10.1523/JNEUROSCI.19-21-09497.1999

Ngo H-V, Fell J, Staresina B (2020) Sleep spindles mediate hippocampal-neocortical coupling during long-duration ripples. eLife 9:e57011. https://doi.org/10.7554/eLife.57011

Norman Y, Raccah O, Liu S, et al (2021) Hippocampal ripples and their coordinated dialogue with the default mode network during recent and remote recollection. Neuron 109:27672780.e5. https://doi.org/10.1016/j.neuron.2021.06.020

Norman Y, Yeagle EM, Khuvis S, et al (2019) Hippocampal sharp-wave ripples linked to visual episodic recollection in humans. Science 365:eaax1030. https://doi.org/10.1126/science.aax 1030

O'Keefe J, Nadel L (1978) The hippocampus as a cognitive map. Clarendon Press ; Oxford University Press, Oxford : New York

O'Keefe J, Recce ML (1993) Phase relationship between hippocampal place units and the EEG theta rhythm. Hippocampus 3:317-330. https://doi.org/10.1002/hipo.450030307

Pacheco Estefan D, Sánchez-Fibla M, Duff A, et al (2019) Coordinated representational reinstatement in the human hippocampus and lateral temporal cortex during episodic memory retrieval. Nat Commun 10:2255. https://doi.org/10.1038/s41467-019-09569-0

Penfield W, Jasper H (1954) Epilepsy and the functional anatomy of the human brain. Little, Brown \& Co., Oxford, England

Penfield W, Roberts L (2014) Speech and Brain Mechanisms. Princeton University Press

Purves D, Augustine GJ, Fitzpatrick D, et al (2001) Excitatory and Inhibitory Postsynaptic Potentials. Neuroscience 2nd edition

Qasim SE, Fried I, Jacobs J (2021) Phase precession in the human hippocampus and entorhinal cortex. Cell 184:3242-3255.e10. https://doi.org/10.1016/j.cell.2021.04.017

Quian Quiroga R (2012) Concept cells: the building blocks of declarative memory functions. Nat Rev Neurosci 13:587-597. https://doi.org/10.1038/nrn3251

Quian Quiroga R, Kraskov A, Koch C, Fried I (2009) Explicit Encoding of Multimodal Percepts by Single Neurons in the Human Brain. Current Biology 19:1308-1313. https://doi.org/10.1016/j.cub.2009.06.060

Quian Quiroga R, Mukamel R, Isham EA, et al (2008) Human single-neuron responses at the threshold of conscious recognition. Proceedings of the National Academy of Sciences 105:3599-3604. https://doi.org/10.1073/pnas.0707043105

Quian Quiroga R, Reddy L, Kreiman G, et al (2005) Invariant visual representation by single neurons in the human brain. Nature 435:1102-1107. https://doi.org/10.1038/nature03687 
Rao VR, Sellers KK, Wallace DL, et al (2018) Direct Electrical Stimulation of Lateral Orbitofrontal Cortex Acutely Improves Mood in Individuals with Symptoms of Depression. Current Biology 28:3893-3902.e4. https://doi.org/10.1016/j.cub.2018.10.026

Ray S, Crone NE, Niebur E, et al (2008) Neural Correlates of High-Gamma Oscillations (60-200 $\mathrm{Hz}$ ) in Macaque Local Field Potentials and Their Potential Implications in Electrocorticography. J Neurosci 28:11526-11536. https://doi.org/10.1523/JNEUROSCI.2848-08.2008

Reddy L, Poncet M, Self MW, et al (2015) Learning of anticipatory responses in single neurons of the human medial temporal lobe. Nat Commun 6:8556. https://doi.org/10.1038/ncomms9556

Reddy L, Self MW, Zoefel B, et al (2021) Theta-phase dependent neuronal coding during sequence learning in human single neurons. Nat Commun 12:4839. https://doi.org/10.1038/s41467-021-25150-0

Reifenstein ET, Kempter R, Schreiber S, et al (2012) Grid cells in rat entorhinal cortex encode physical space with independent firing fields and phase precession at the single-trial level. PNAS 109:6301-6306. https://doi.org/10.1073/pnas.1109599109

Ritchey M, Wing EA, LaBar KS, Cabeza R (2013) Neural Similarity Between Encoding and Retrieval is Related to Memory Via Hippocampal Interactions. Cerebral Cortex 23:28182828. https://doi.org/10.1093/cercor/bhs258

Romanelli P, Piangerelli M, Ratel D, et al (2018) A novel neural prosthesis providing long-term electrocorticography recording and cortical stimulation for epilepsy and brain-computer interface. Journal of Neurosurgery 130:1166-1179. https://doi.org/10.3171/2017.10.JNS17400

Romanska A, Rezlescu C, Susilo T, et al (2015) High-Frequency Transcranial Random Noise Stimulation Enhances Perception of Facial Identity. Cerebral Cortex 25:4334-4340. https://doi.org/10.1093/cercor/bhv016

Rutishauser U, Aflalo T, Rosario ER, et al (2018) Single-Neuron Representation of Memory Strength and Recognition Confidence in Left Human Posterior Parietal Cortex. Neuron 97:209-220.e3. https://doi.org/10.1016/j.neuron.2017.11.029

Rutishauser U, Mamelak AN, Schuman EM (2006) Single-Trial Learning of Novel Stimuli by Individual Neurons of the Human Hippocampus-Amygdala Complex. Neuron 49:805813. https://doi.org/10.1016/j.neuron.2006.02.015

Rutishauser U, Ye S, Koroma M, et al (2015) Representation of retrieval confidence by single neurons in the human medial temporal lobe. Nat Neurosci 18:1041-1050. https://doi.org/10.1038/nn.4041

Sakon JJ, Kahana MJ (2021) Hippocampal ripples signal contextually-mediated episodic recall

Sauseng P, Klimesch W, Heise KF, et al (2009) Brain Oscillatory Substrates of Visual ShortTerm Memory Capacity. Current Biology 19:1846-1852. https://doi.org/10.1016/j.cub.2009.08.062

Serre T, Oliva A, Poggio T (2007) A feedforward architecture accounts for rapid categorization. Proceedings of the National Academy of Sciences 104:6424-6429. https://doi.org/10.1073/pnas.0700622104

Silvanto J, Muggleton N, Walsh V (2008) State-dependency in brain stimulation studies of perception and cognition. Trends in Cognitive Sciences 12:447-454. https://doi.org/10.1016/j.tics.2008.09.004 
Skelin I, Zhang H, Zheng J, et al (2021) Coupling between slow waves and sharp-wave ripples engages distributed neural activity during sleep in humans. PNAS 118:. https://doi.org/10.1073/pnas.2012075118

Song Y, Shi S, Li J, Zhang H (2018) Directional Skip-Gram: Explicitly Distinguishing Left and Right Context for Word Embeddings. In: Proceedings of the 2018 Conference of the North American Chapter of the Association for Computational Linguistics: Human Language Technologies, Volume 2 (Short Papers). Association for Computational Linguistics, New Orleans, Louisiana, pp 175-180

Staresina BP, Henson RNA, Kriegeskorte N, Alink A (2012) Episodic Reinstatement in the Medial Temporal Lobe. Journal of Neuroscience 32:18150-18156. https://doi.org/10.1523/JNEUROSCI.4156-12.2012

Staresina BP, Michelmann S, Bonnefond M, et al (2016) Hippocampal pattern completion is linked to gamma power increases and alpha power decreases during recollection. eLife 5:e17397. https://doi.org/10.7554/eLife.17397

Suthana N, Aghajan ZM, Mankin EA, Lin A (2018) Reporting guidelines and issues to consider for using intracranial brain stimulation in studies of human declarative memory. Frontiers in neuroscience 905

Suthana N, Haneef Z, Stern J, et al (2012) Memory Enhancement and Deep-Brain Stimulation of the Entorhinal Area. New England Journal of Medicine 366:502-510. https://doi.org/10.1056/NEJMoa1107212

Tsao A, Sugar J, Lu L, et al (2018) Integrating time from experience in the lateral entorhinal cortex. Nature 561:57-62. https://doi.org/10.1038/s41586-018-0459-6

Tulving E (2002) Episodic Memory: From Mind to Brain. Annual Review of Psychology 53:125. https://doi.org/10.1146/annurev.psych.53.100901.135114

Umbach G, Kantak P, Jacobs J, et al (2020) Time cells in the human hippocampus and entorhinal cortex support episodic memory. PNAS 117:28463-28474. https://doi.org/10.1073/pnas.2013250117

Vaz AP, Inati SK, Brunel N, Zaghloul KA (2019) Coupled ripple oscillations between the medial temporal lobe and neocortex retrieve human memory. Science 363:975-978. https://doi.org/10.1126/science.aau8956

Voss JL, Federmeier KD (2011) FN400 potentials are functionally identical to N400 potentials and reflect semantic processing during recognition testing. Psychophysiology 48:532546. https://doi.org/10.1111/j.1469-8986.2010.01085.x

Watrous AJ, Lee DJ, Izadi A, et al (2013) A comparative study of human and rat hippocampal low frequency oscillations during spatial navigation. Hippocampus 23:656-661. https://doi.org/10.1002/hipo.22124

Wilson MA, McNaughton BL (1994) Reactivation of Hippocampal Ensemble Memories During Sleep. Science 265:676-679. https://doi.org/10.1126/science.8036517

Woodruff CC, Hayama HR, Rugg MD (2006) Electrophysiological dissociation of the neural correlates of recollection and familiarity. Brain Research 1100:125-135. https://doi.org/10.1016/j.brainres.2006.05.019

Xiao X, Dong Q, Gao J, et al (2017) Transformed Neural Pattern Reinstatement during Episodic Memory Retrieval. J Neurosci 37:2986-2998. https://doi.org/10.1523/JNEUROSCI.2324-16.2017

Xue G, Juan C-H, Chang C-F, et al (2012) Lateral prefrontal cortex contributes to maladaptive decisions. PNAS 109:4401-4406. https://doi.org/10.1073/pnas.1111927109 
Yaffe RB, Kerr MSD, Damera S, et al (2014) Reinstatement of distributed cortical oscillations occurs with precise spatiotemporal dynamics during successful memory retrieval. PNAS 111:18727-18732. https://doi.org/10.1073/pnas.1417017112

Yanagisawa T, Hirata M, Saitoh Y, et al (2011) Real-time control of a prosthetic hand using human electrocorticography signals. J Neurosurg 114:1715-1722. https://doi.org/10.3171/2011.1.JNS101421

Zeldenrust F, Wadman WJ, Englitz B (2018) Neural Coding With Bursts - Current State and Future Perspectives. Frontiers in Computational Neuroscience 12:48. https://doi.org/10.3389/fncom.2018.00048

Zhang H, Fell J, Axmacher N (2018) Electrophysiological mechanisms of human memory consolidation. Nat Commun 9:4103. https://doi.org/10.1038/s41467-018-06553-y

Zheng J, Gómez Palacio Schjetnan A, Yebra M, et al (2021) Cognitive boundary signals in the human medial temporal lobe shape episodic memory representation. Neuroscience 